\title{
A Hybrid System for Dental Milling parameters optimisation
}

\author{
Vicente Vera ${ }^{1}$, Javier Sedano ${ }^{2}$, Emilio Corchado ${ }^{3}$, Raquel Redondo ${ }^{4}$, Beatriz \\ Hernando $^{1}$, Monica Camara ${ }^{2}$, Amer Laham ${ }^{3}$, Alvaro Enrique Garcia ${ }^{1}$ \\ ${ }^{1}$ Facultad de Odontología, UCM, Madrid, Spain \\ \{vicentevera, aegarcia\}@odon.ucm.es \\ ${ }^{2}$ Dept. of A.I. \& Applied Electronics, Castilla y León Technological Institute, Burgos, Spain \\ \{javier.sedano, monica.camara\}@itcl.es \\ ${ }^{3}$ Departamento de Informática y Automática, Universidad de Salamanca, Salamanca, Spain \\ escorchado@usal.es \\ ${ }^{4}$ Department of Civil Engineering, University of Burgos, Burgos, Spain \\ rredondo@ubu.es
}

\begin{abstract}
This study presents a novel hybrid intelligent system which focuses on the optimisation of machine parameters for dental milling purposes Based on the following phases. Firstly, an unsupervised neural model extracts the internal structure of a data set describing the model and also the relevant features of the data set which represents the system. Secondly, the dynamic system performance of different variables is specifically modelled using a supervised neural model and identification techniques from relevant features of the data set. This model constitutes the goal function of the production process. Finally, a genetic algorithm is used to optimise the machine parameters from a non parametric fitness function. The reliability of the proposed novel hybrid system is validated with a real industrial use case, based on the optimisation of a highprecision machining centre with five axes for dental milling purposes.
\end{abstract}

\section{Introduction}

The optimisation process of machine parameters could significantly help to increase companies' efficiencies and substantially contributes to costs reductions in preparation and setting machines processes and it also helps in the production process using new materials.

Nevertheless, the variables and parameters setting processes are a well-known problem that has not been fully resolved yet. Several different techniques are proposed in the literature. In [1], is used a Taguchi orthogonal array to optimise effect of injection parameters. In [2] the influence of operating parameters of ultrasonic machining is studied using Taguchi and F-test method. In [3] is researched as to 
improve the quality of the $\mathrm{KrF}$ excimer laser micromachining of metal using the orthogonal array-based experimental design method.

Conventional methods can be greatly improved through the application of soft computing techniques [4].

The novel proposed method was tested and validated using a four-step procedure based on several soft computing techniques as artificial neural networks (ANN) and genetic algorithms (GA). Firstly, the dataset is analysed using projection methods such as Principal Component Analysis (PCA) [5], [6], [7] and Cooperative Maximum-Likelihood Hebbian Learning (CMLHL)[8] to analyse the internal structure of the dataset to establish whether the data set is sufficiently informative. Then those methods are applied to perform feature selection as a pre-processing step. It means that if the initial collected data set, once analysed shows a certain degree of clustering, it can be seen as a sign of a representative data set (this means that there is not a single problem related to any sensor when collecting the information and the process is well defined by such data set. Then, the following steps of the process can be applied. And thus the most representative features are identified and used in the following steps. At this phase, a model is generated during the modelling stage to estimate production time errors by modelling techniques. Finally, the ANN model obtained in the last step is used as fitness function to be optimised in the genetic algorithm.

The rest of this paper is organised as follows. Section 2 introduces the unsupervised neural models for analysing the internal structure of the data sets and to perform feature selection.. Section 3 deals with system identification techniques used in the system modelling. Section 4 introduces the applied GA. Section 5 describes the real industrial use case. The final section presents the different models that are used to solve the high precision dental milling optimisation use case. Finally conclusions are set out and some comments on future research lines are outlined.

\section{Soft Computing for Data Structure Analysis}

Soft Computing is a set of several technologies whose aim is to solve inexact and complex problems [9] It investigates, simulates, and analyses very complex issues and phenomena in order to solve real-world problems [10]. Soft Computing has been successfully applied in feature selection, and plenty of algorithms are reported in the literature [11], [12], [13].

Feature Selection and extraction [14], [15] entails feature construction, space dimensionality reduction, sparse representations and feature selection among others. They are all commonly used pre-processing tools in machine learning tasks, which include pattern recognition. Although researchers have grappled with such problems for many years, renewed interest has recently surfaced in feature extraction. 
In this research, an extension of a neural PCA version [5], [6], [7] and other extensions are used to study the internal structure in the data set as well as to select the most relevant input features for feature selections porpuses.

Then, this research uses the feature selection approach based on the dimension reduction issue. Initially, some projection methods as PCA [5], [6], [7], MLHL [16] and CMLHL [8] are applied. In a first step they aim to analyse the internal structure of a representative data set of a real use case. If after applying these models, a clear internal structure can be identified, this means that the data recorded is informative enough. Otherwise, data must be properly collected again [17], [18].

\section{System Modelling Using Identification Algorithms}

System identification (SI) [19] aims to obtain mathematical models to estimate the behaviours of a physical process whose dynamic equations are unknown. The identification criterion consists in evaluating the group of candidate models that best describes the dataset gathered for the experiment. The goal is to obtain a model that meets the following premise [19]: a good model is one that makes good predictions and which produces small errors when the observed data is applied.

Classic SI refers to the parametrical literature, which has its origin in the linear system analysis [20]. Nevertheless, increased computational capability and the availability of soft computing techniques have widened research into SI. ANNs are one of the most interesting soft computing paradigms used in SI.

The SI procedure comprises several steps [19], [21]: the selection of the models and their structure, the learning methods [22], [23], [20], the identification and optimisation criteria and the validation method. Validation ensures that the selected model meets the necessary conditions for estimation and prediction. Typically, validation is carried out using three different methods: the residual analysis -by means of a correlation test between inputs, their residuals and their combinations-; the mean squared error (MSE) and the generalisation error value -normalised sum of squared errors (NSSE) - and finally a graphical comparison between the desired outputs and the model outcomes through simulation [20], [17], [18].

\section{Genetic Algorithm for System Optimisation}

Metaheuristic algorithms are considered as a computational method that optimises a problem by iteratively trying to improve a candidate solution with regard to a given measure of quality. Metaheuristics are more effective and specialised than the classical heuristics. They combine more exclusive neighbourhood search, memory structures and recombination of solutions and tend to provide better results. However, their running time is unknown and they are usually more time consuming than the classical heuristics. Metaheuristics make few or no assumptions about the problem 
being optimised and can search very large spaces of candidate solutions. Within these algorithms, there are two well-known types among others, such as the genetic algorithms [24], and the simulated annealing algorithm [25].

GA are adaptive heuristic search algorithm that mimics the process of natural evolution -Darwin's theory about evolution-. This heuristic is routinely used to generate useful solutions to optimisation and search problems. It solves both constrained and unconstrained optimisation problems. GA is a method for moving from one population of "chromosomes" to a new population by using a kind of "natural selection" together with the genetics, inspired operators of crossover, mutation and inversion. In the literature are found a large number of examples [26], [27], [28], [29], [30]

\section{A High Precision Industrial Use Case Scenario}

This study describes the way in which a hybrid artificial intelligent system can be applied to improve the last step of a high precision industrial system for the manufacture of metal dental pieces, by optimising the time error detection for dental milling process, as shown in Figure 1.

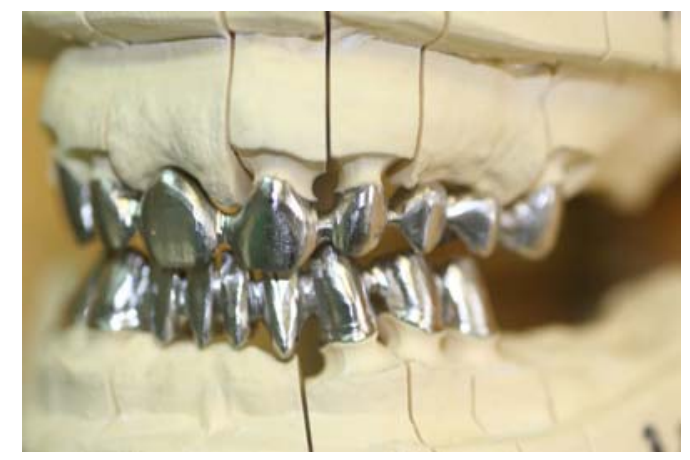

Fig. 1. Metal pieces manufactured by a dynamic high-precision machining centre with five axes

A dynamic high-precision machining centre with five axes was applied in this research. This real industrial use case is described by an initial data set of 109 samples obtained by a dental scanner in the manufacturing of dental pieces with a toric tool characterized by 7 input variables (Radius, Number of pieces, Thickness, Revolutions, Feed rate $\mathrm{X}, \mathrm{Y}$ and Z) and 1 output variable -Real time of work- as shown in Table 1. Time errors for manufacturing are the difference between the estimated time by the machine itself and real production time -negative values indicates that real exceeds estimated time-. 
Table 1. Values of each variable used in the process

\begin{tabular}{ll}
\hline Variable (Units) & Range of values \\
\hline Radius (mm.) & 0.25 to 2 \\
Number of pieces & 1 to 4 \\
Thickness (mm.) & 8 to 18 \\
Revolutions per minute (RPM) & 10,00 to 38,000 \\
Feed rate X & 75 to 3,000 \\
Feed rate Y & 75 to 3,000 \\
Feed rate Z & 75 to 2,000 \\
Real time of work (s.) & 81 to 1,924 \\
Time errors for manufacturing (s.) & -3 to -332 \\
\hline
\end{tabular}

\section{Optimising a Real Dental Milling Process}

The manufacturing of dental pieces process optimisation in terms of time errors based on the optimisation of the system behaviour is carried out by means of an ANN estimated model. Firstly, the dental manufacturing process is parameterised and its dynamic performance in normal operation is obtained by the real manufacturing of dental pieces. Then, the gathered data is processed using CMLHL to identify internal data set structures in order to determine the ability of the data set to be modelled and to identify the most relevant features. This allows a third step, knowing a priori, that the model to be obtained can be achieved.

Once the model has been obtained -in the third step-, it is then used as a reference model and also as fitness functions in a GA. The GA calculates the best conditions under normal operating conditions in a dental milling process for manufacturing dental pieces, so if the operator wants to make a dental piece, the best machining conditions might be determined to minimize manufacturing time errors compared to the estimated manufacturing time which is given by the machine itself.

This section deals with the description of each step once the data set is collected (see Section 4). In the next subsection, the generation of the data set which will be used in the process is described. Sub-Section 6.1 presents the PCA and CMLHL steps, in Sub-Section 6.2 the procedure to obtain the time error model is detailed, while in Sub-Section 6.3 the GA is applied.

\section{Identification of the Relevant Features}

PCA and CMLHL are techniques for identifying the internal structure of a data set and also to identify the most relevant variables, as detailed in Section 2. Both of which were applied to this real industrial use case. Then, by means of projection methods it is analysed whether the data set is sufficiently representative of a case 
study, and the most relevant variables are identified to reduce the computational cost in the third step.

\section{Modelling a Normal Dental Milling Operation}

Once the relevant variables and their transformations have been extracted from the production data, then a model to fit the normal manufacturing operation should be obtained. This is done to identify bias in the estimated production time, which, in the end, is used as fitness function -time error in the manufacturing of dental pieces-. The different model learning methods used in this study were implemented in Matlab $\odot$ [31].

Moreover, several different indexes were used to validate the models [17], [18] such as the percentage representation of the estimated model; the oranhiral representation for the prediction - $\hat{y}_{1}(t \mid m)$ - versus the measured output - $\hat{y}_{1}(t)$-; the loss function or error function $(\mathrm{V})$ and the generalization error value.

The percentage representation of the estimated model is calculated as the normalised mean error for the prediction (FIT1). The loss function or error function $(\mathrm{V})$ is the numeric value of the mean square error (MSE) that is computed with the estimation data set. Finally, the generalisation error value is the numeric value of the normalised sum of square errors (NSSE) that is computed alongside with the validation data set (NSSE1) and with the test data set (NSSE2) [32].

\section{Optimisation of a Normal Dental Milling Operation}

In this case study of dental pieces manufacturing, GAs are concerned with obtaining variables that best optimised the time errors. Firstly this process of optimisation is started with a set of solutions randomly called population -chromosomes-. Then, each individual in the population is evaluated by the fitness function obtained in the last step -ANN model of the manufacturing system-. GA, so as the different types of genetic operators -selection, crossover, mutation- used in this study were implemented in Matlab®.

\section{Experiment and Results}

The real industrial use case was analysed in order to select the features that best describe the relationships with manufacturing time errors.

CMLHL is a powerful technique for identifying internal dataset structures. The axes forming the projections (Figure 2.a and Figure2.b) represent combinations of the variables contained in the original datasets. In the case of PCA, the model is looking for those directions with the biggest variance, when CMLHL is looking for those which measure how interesting is a dimension/direction. In this case, those are the directions which are as less Gaussian as possible, (by analysing the kurtosis) [8], [16]. 


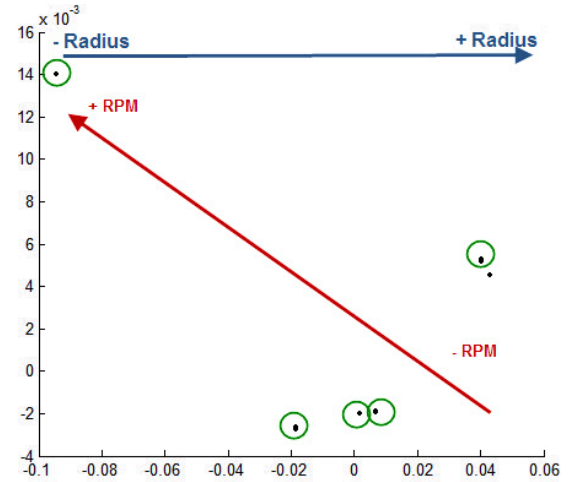

Fig.2.a. Projection of PCA

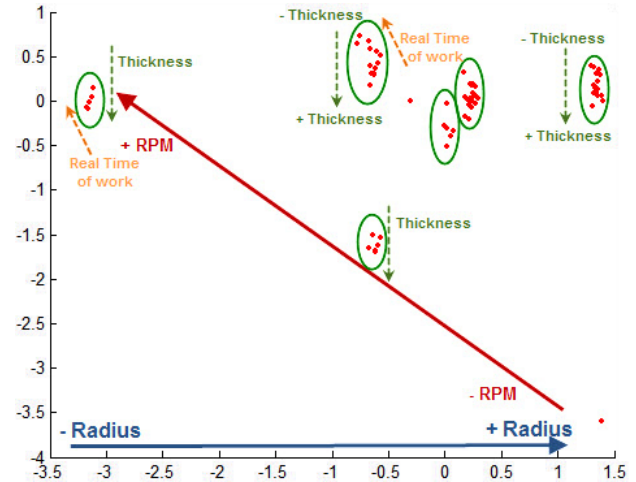

Fig.2.b. CMLHL projections

Fig. 2. PCA projections (Figure 2.a) and CMLHL projections (Figure 2.b)

As seen in Figure 2, PCA (Figure 2.a) and CMLHL (Figure 2.b), both found a clear internal structure in the dataset. Both methods identified 'radius' and 'RPM' variables as the relevant ones. CMLHL projection gives more information since it recognises the 'thickness' as another important variable and also 'real time of work'. CMLHL provides a more sparse representation than the PCA model.

An analysis of the results obtained with the CMLHL model, (Figure 2.b), leads to the conclusion that it has identified several different clusters ordered by 'radius' and 'RPM' variables.

Inside each cluster (Figure 2.b), there are further classifications by 'thickness' and 'real time of work'. The dataset can be said to have an interesting internal structure.

When the dataset is considered sufficiently informative, as in this case, the step for modelling the relations between inputs and production time errors in the process begins, through the application of several conventional ANN modelling systems.

Thus, an ANN was used to monitor time error detection in the manufacturing of dental pieces by using the pre-processed data set from the input and output normalisation step -zero mean and unity standard deviation-, the reduction of the input vectors dimension -the data set gathered in the previous step- and the use of early stopping and Bayesian regularization techniques [33].

The model was obtained using the Bayesian regularised criterion when the ANN is determined the last step starts and it is then used as fitness functions in a GA. The ANN structure -Feedforward Network- has 25 hyperbolic tangent units -layer 1-, 20 hidden hyperbolic tangent units -layer 2-, 4 hidden hyperbolic tangent units -layer 3and 1 linear output unit. The network is estimated using the Lenvenberg-Marquardt method. Indexes of the model are FIT1: 80.1\%, V: 0.043.and NSSE1: 0.031.

The fitness function is the model of the time error in the dental pieces manufacturing. GA starts with a randomly generated initial population of size 60 individuals. Tournament selection is used to determine the parents for the next generation. Individuals from the current population are selected proportionally to their 
fitness and forming in this way the basis for the next generation. Two-point crossover combines two parents to form a new individual for the next generation and uniform mutation with a 0.01 rate makes small changes in the individuals in the population. The population obtained by these genetic modifications is evaluated against the fitness function and enters a new search process in the next generation. The algorithm stops after fixed number of generations is reached and the best individual is returned as a solution to the given problem.

Figure 3 shows the output response of the time error for different unnormalised input variable ranges. In Figure 3.a the $\mathrm{X}$-axis shows the thickness, from $8 \mathrm{~mm}$ to 18 $\mathrm{mm}$., the Y-axis shows the revolutions per minute, from 10,000 to 38,000 in RPM. and the Z-axis represents the unnormalised output variable range from -400 to 0 in $\mathrm{s}$. for a constant value of a radius of $1,5 \mathrm{~mm}$. The time error is shown on the bar, too. In Figure 3.b the $\mathrm{X}$-axis shows the thickness from 8 to $18 \mathrm{in} \mathrm{mm}$, the $\mathrm{Y}$-axis shows the radius, from 0.25 to 2 in $\mathrm{mm}$ and the $\mathrm{Z}$-axis represents the unnormalised output variable range from -250 to 0 in $\mathrm{s}$. for a constant value of revolutions per minute of 22,000 RPM. The time error is shown on the bar, too. The time error can be optimised for different values of radius, thickness and revolutions per minute; i.e., it is possible to achieve the less time error and to find the optimal value of the revolutions per minute for a thickness of $14 \mathrm{~mm}$. and a radius of $1.5 \mathrm{~mm}$. -both values fixed- in this case the time error and the revolutions per minute are -38.4 s. and 38,000 RPM., respectively. Also if the radius and the time error are fixed to $1.5 \mathrm{~mm}$. and $-100 \mathrm{~s}$., respectively, the thickness and the revolutions to optimise those variables are 12.41 mm. and 19,900 RPM.

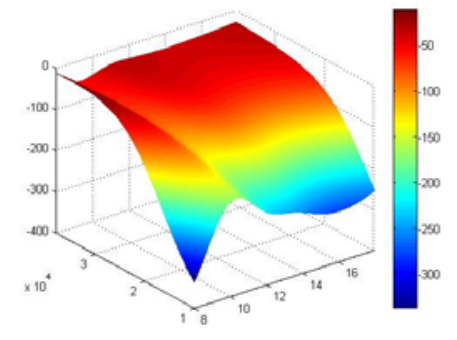

Fig.3.a. 3D graph, the $X$-axis represents the thickness, the Y-axis the RPM. and the Zaxis the output -time error- for a constant value of radius of $1,5 \mathrm{~mm}$.

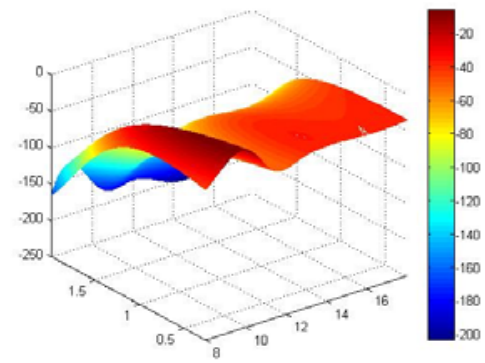

Fig.3.b. 3D graph, the $\mathrm{X}$-axis represents the thickness, the Y-axis the radius, and the Zaxis the output -time error- for a constant value of 22,000 RPM.

Fig. 3. Output response of the time error for different unnormalised input variable ranges

\section{Conclusions and Future Work}

The novel hybrid artificial intelligence system for the optimisation of this industrial process can be used in the optimisation of machine parameters for industrial processes 
in general. The process increases the companies' efficiency and substantially contributes to costs reduction of preparation and setting machine processes and it also helps in the production process using new materials. This method has been used in this case for the optimisation and adjustments during the manufacturing process of dental pieces such as implants according to medical specifications of precisely moulded.

The dental milling presents an important time error rate of manufacturing which is about $48 \%$. This is due to the difference between the estimated time by the machine itself and the real production. The model obtained is capable of modelling more than $80 \%$ of the actual measurements in relation to time error -modelling more than $90 \%$ of the real time of work-. This helps to reduce the error and the variability rate of manufacturing processes down to $10 \%$, compared to $48 \%$ initially (Acceptable error rate in planning work for dental milling).

Future lines of research include modelling the temperature and the erosion (errors in length or tooth wear), which is a measure of the quality of the dental milling process. Finally, an algorithm will be developed to automatically identify the best operating conditions: minor time errors for the manufacturing of dental pieces and minor erosion. The resulting model would moreover be applied to different metals used in prosthetic dentistry and in other industrial processes.

Acknowledgments. This research is partially supported through projects of the Spanish Ministry of Science and Innovation TIN2010-21272-C02-01, and PID 560300-2009-11], the Spanish Ministry of Science and Technology TIN2008-06681C06-04] and Junta de Castilla y Leon exp. CCTT/10/BU/0002. The authors would also like to thank the vehicle interior manufacturer, Grupo Antolin Ingenieria, S.A., within the framework of project MAGNO2008 - 1028.- CENIT also funded by the Spanish Ministry of Science and Technology and also to ESTUDIO PREVIO (Madrid-Spain) for its collaboration in this research.

\section{References}

1. Ozcelik B, Ozbay A, Demirbas E (Nov 2010) Influence of injection parameters and mold materials on mechanical properties of ABS in plastic injection molding. International Communications in Heat and Mass transfer, 37(9):1359-1365

2. Kumar V, Jaimal Singh Khamba (Jan 2008) Statistical analysis of experimental parameters in ultrasonic machining of tungsten carbide using the Taguchi approach. Journal of the American Ceramic Society, 91(1):92-96

3. Li J, Ananthasuresh GK (Jan 2001) A quality study on the excimer laser micromachining of electro-thermal-compliant micro devices. Journal of Micromechanics and microengineering, 11(1):38-47

4. Hsu-Hwa Chang, Yan-Kwang Chen (Jan 2011) Neuro-genetic approach to optimize parameter design of dynamic multiresponse experiments. Applied Soft Computing, 11(1):436-442 
5. Pearson K (1901) On lines and planes of closest fit to systems of points in space. Philosophical Magazine, 2(6):559-572

6. Hotelling H (1933) Analysis of a complex of statistical variables into Principal Components. Journal of Education Psychology, 24:417-444

7. Oja E, Ogawa H, Wangviwattana J (May 1992) Principal Components Analysis by Homogeneous Neural Networks, part 1, The Weighted Subspace Criterion, IEICE Transaction on Information and Systems, E75D: 366-375

8. Corchado E, Fyfe C (2003) Connectionist Techniques for the Identification and Suppression of Interfering Underlying Factors. Int. Journal of Pattern Recognition and Artificial Intelligence, 17(8), pp. 1447-1466.

9. Kohonen T (1998) The self-organizing map. Neurocomputing 21(1-3): 1-6

10. Sedano J, de la Cal E, Curiel L, Villar J.R., Corchado E (2009) Soft Computing for detecting thermal insulation failures in buildings. Proceedings of the 9thInternational Conference on Computational and Mathematical Methods in Science and Engineering, CMMSE2009, Gijon, Spain.

11. Leray P, Gallinari P (January 1999) Feature selection with neural networks. Behaviormetrika, Vol. 26

12. Verikas A, Bacauskiene M (September 2002) Feature selection with neural networks . Pattern Recognition Letters. Volume 23, Issue 11, Pages 1323-1335

13. Diaconis P, Freedman D (1984) Asymptotics of Graphical Projections. The Annals of Statistics. 12(3): 793-815

14. Guyon I, Elisseeff A (2003) An introduction to variable and feature selection. Journal of Machine Learning Research, Special Issue on variable and Feature Selection, 3:1157-1182

15. Liu H, Yu L (2005) Toward integrating feature selection algorithms for classification and clustering. IEEE Knowledge and Data Engineering, IEEE Transactions, 17(4):491-502

16. Corchado E, MacDonald D, Fyfe C (2004) Maximum and Minimum Likelihood Hebbian Learning for Exploratory Projection Pursuit. Data Mining and Knowledge Discovery, 8(3):203-225

17. Vera V, Corchado E, Redondo R, Sedano J et al. (2010) Optimizing a dental milling process by means of Soft Computing Techniques.10th International Conference on Intelligent Systems Design and Applications.978-1-4244-8136-1/10/\$26.00 IEEE. Pages 1430-1435

18. Vera V, Corchado E, Redondo R, Sedano J et al. (2010) A bio-inspired computational highprecision dental milling system. Proceedings of the World Congress on Nature and Biologically Inspired Computing (NaBIC2010) 978-1-4244-7375-5/10/\$26.00 2010 IEEE pages $430-436$

19. Ljung L. System Identification. Theory for the User. Prentice-Hall, Upper Saddle River, N.J., USA, 2nd edition.

20. Sedano J, Corchado E, Curiel L et al. (2009) The application of a two-step AI model to an automated pneumatic drilling process, International Journal of Computer Mathematics, Vol. 86, No. 10-11, pp. 1769-1777

21. Nørgaard M, Ravn O, Poulsen N.K., Hansen L.K. (2000) Neural Networks for Modelling and Control of Dynamic Systems. Springer-Verlag, London U.K.

22. Stoica P, Söderström T (1982) A useful parametrization for optimal experimental design. In IEEE Trans. Automatic. Control, AC-27 
23. He X, Asada H (1993) A new method for identifying orders of input-output models for nonlinear dynamic systems. In Proc. of the American Control Conf., S.F., pp. 2520-2523, California

24. Hayes-Roth F (Aug 1975) Review of "adaptation in natural and artificial systems by john h. holland", the u. of michigan press, 1975. SIGART Bull., pages 15-15.

25. Kirkpatrick S, Gelatt Jr. C.D., Vecchi M.P (1983) Optimization by simulated annealing. Science, 220:671-680.

26. Aliev R.A., Aliev R.R., Guirimov B., Uyar K (June 2008) Dynamic data mining technique for rules extraction in a process of battery charging. Appl. Soft Comput., pages 1252-1258

27. Chaudhry I, Drake P (2009) Minimizing total tardiness for the machine scheduling and worker assignment problems in identical parallel machines using genetic algorithms. The International Journal of Advanced Manufacturing Technology, 42:581-594. 10.1007/s00170-008-1617-z.

28. Fujita S (Nov 2009) Retrieval parameter optimization using genetic algorithms. Inf. Process. Manage., 45:664-682

29. Oliveira A.L.I., Braga P.L., Lima R.M.F, Cornélio M.L (Nov 2010) Ga-based method for feature selection and parameters optimization for machine learning regression applied to software effort estimation. Inf. Softw. Technol., 52:1155-1166.

30. Palanisamy P, Rajendran I, Shanmugasundaram S (2007) Optimization of machining parameters using genetic algorithm and experimental validation for end-milling operations. The International Journal of Advanced Manufacturing Technology, 32:644-655. 10.1007/s00170-005-0384-3.

31. Demuth H, Beale M, Hagan M (2010) Neural Network Toolbox User's Guide, The Mathworks, Inc.

32. Sedano J, Corchado E, Curiel L, Villar J. R, de la Cal E (2010) Detection of heat flux failures in building using a soft computing diagnostic system, Neural network world 20(7) pp.883-898.

33. Mackay D.J.C. (1992) “Bayesian interpolation”, Neural Computation, Vol. 4, No. 3, pp. 415-447. 